\title{
Influence of crosslinker and ionic comonomer concentration on glass transition and demixing/mixing transition of copolymers poly $(N$-isopropylacrylamide) and poly(sodium acrylate) hydrogels
}

\author{
Iwona Zarzyka • Marek Pyda • Maria Laura Di Lorenzo
}

Received: 14 August 2013 /Revised: 4 October 2013 / Accepted: 7 October 2013 /Published online: 27 October 2013

(C) The Author(s) 2013. This article is published with open access at Springerlink.com

\begin{abstract}
Hydrogels based on $N$-isopropylacrylamide and sodium acrylate as ionic comonomer were synthesized by free radical polymerization in water using $N, N^{\prime}-$ methylenebisacrylamide as crosslinker and ammonium persulfate as initiator. The glass transition of dried copolymers poly( $N$-isopropylacrylamide) (PNIPA) and poly(sodium acrylate) (SA) gels and demixing/mixing transition of PNIPA-SA hydrogels swollen with increasing amounts of water were studied using conventional differential scanning calorimetry. In the crosslinked polymers, the glass transition linearly increases, and the transition range becomes broader, with increasing crosslinker content. Increasing content of ionic comonomer also produces an increase of glass transition temperature, which moves to higher temperatures with higher sodium acrylate fraction. The influence of chemical structure of PNIPA-SA hydrogels on the lower critical solution temperature (LCST) of PNIPA-SA/water mixtures during heating and cooling was quantified as function of the content of the crosslinker and the ionic comonomer, as well as water content of the hydrogel in the range from 95 to $70 \mathrm{wt} \%$. At parity of water content, the LCST occurs at higher temperatures for gels containing higher amounts of sodium acrylate. Similarly, the introduction of $N, N^{\prime}-$ methylenebisacrylamide causes an increase of the LCST, which grows with increasing of crosslinking degree of the hydrogel.
\end{abstract}

\footnotetext{
I. Zarzyka $\cdot$ M. Pyda $(\bowtie)$

Department of Chemistry, Rzeszow University of Technology, 35-959 Rzeszow, Poland

e-mail:mpyda@utk.edu

\section{L. Di Lorenzo}

Consiglio Nazionale delle Ricerche, Istituto di Chimica e Tecnologia dei Polimeri, c/o Compresorio Olivetti, Via Campi Flegrei 34, 80078 Pozzuoli, NA, Italy
}

Keywords Hydrogels · Copolymer .

Poly $(N$-isopropylacrylamide $) \cdot$ Demixing $\cdot$ LCST

\section{Introduction}

The liquid-liquid phase diagram at constant pressure of polymer solutions is usually determined by plotting the temperature of incipient phase separation (cloud point) as function of polymer concentration. The minimum in the cloud point vs. concentration curve is usually named lower critical solution temperature (LCST), since it denotes the limit of phase separation between polymer and solvent. A number of thermosensitive polymers, like poly $(N$-substituted acrylamides) [1-4], poly( $N$-vinylisobutyramide) [5], or poly(vinylmethyl ether) [6], are characterized by a LCST when dissolved in aqueous media.

At temperatures below the LCST, polymer-water mixture is a one-phase system. Phase separation is connected with the interactions between polymer chains and water molecules, as well as with chain-chain effects. Below the phase transition temperature, the macromolecule is maintained in solution, owing to the creation of hydrogen bonds between hydrophilic fragments of the polymer chain and the water molecules. Heating the system results in destruction of the solvate sphere, hydrophobic effects become dominant, which leads to macroscopic volume-phase transition-demixing $[1,7]$.

The possibility of controlling the LCST forms the basis for utilizing stimuli-sensitive polymers. The critical solution temperature can be changed by controlling the content and structure of the macromolecule. The LCST depends on the content of hydrophilic and hydrophobic elements in the macromolecule and on their distribution (random or block copolymer), as well as on the 
macromolecule topology (linear, graft, and hyperbranched polymer) and their molecular mass. The changes taking place under the influence of the temperature within the polymer phase can be utilized in many branches of contemporary chemistry, technology, and biochemistry, e.g., for hyperthermal medicine releasing, for selective protein bioseparating, smart bioactive carriers, or metal carriers in catalysis [7-12]. Thermosensitive polymers are also utilized in the separation processes of protein solutions and of other macromolecular, biologically active substances $[2,13,14]$, as well as in the absorption of heavy metals [15].

The most commonly used monomer for the synthesis of thermosensitive polymers is $N$-isopropylacrylamide (PNIPA), which can form a hydrogel upon addition of proper crosslinking agents and immersion in water. During the latest years, the number of publication discussing the synthesis, characteristics, and uses of the thermosensitive hydrogels based on PNIPA, is steadily growing $[1,10,16,17]$. Similarly to other polymer gels, consisting of a crosslinked polymer network immersed in liquid, PNIPA hydrogel undergoes a volume-phase transition: when external conditions such as temperature, solvent composition, or osmotic pressure are changed, the gel reversibly swells or shrinks, with a discontinuous volume variation [18].

PNIPA undergoes a fast and reversible coil-to-globule transition around its LCST in aqueous media [19]. At temperatures below the LCST, PNIPA chains are hydrated and adopt flexible and expanded random coil conformations in water. Above the LCST, PNIPA chains become dehydrated and collapse into a tightly packed globular conformation. Since the transition temperature is around $30-35{ }^{\circ} \mathrm{C}[1,20]$, PNIPA hydrogel is an excellent candidate for biochemical systems, like drug delivery systems, because the effective release of drugs is controlled by temperature-sensitive swelling characteristics of drug incorporating PNIPA hydrogel [21].

Unfortunately, water absorption capacity of the PNIPA gel is limited. Incorporation of hydrophilic/hydrophobic comonomers into PNIPA hydrogels can modify the swelling behaviors as well as other physicochemical properties, including mechanical strength and solute diffusivity. For higher water absorption capacity, PNIPA can be copolymerized with functional monomers such as acrylic acid or other ionic comonomers [22-25].

The present work focuses on investigation of the thermal properties of PNIPA-based hydrogels, where sodium acrylate (SA) is used as ionic comonomer and $N, N^{\prime}$ methylenebisacrylamide (BIS) is added as crosslinker. The influence of the chemical structure of PNIPA hydrogel on $T g$ and LCST of PNIPA/water mixtures upon heating and cooling is quantified as function of crosslinker and ionic comonomer content.

\section{Experimental part}

Materials

$N$-isopropylacrylamide (NIPA) $97 \%, N, N, N^{\prime}, N^{\prime}-$ tetramethylethylenediamine (TEMED) $99 \%$ and sodium acrylate (SA) $97 \%$ were purchased from Sigma-Aldrich. $N$, $N^{\prime}$-methylenebisacrylamide $\geq 99.5 \%$ was provided by Fluka. Ammonium persulfate (APS) $98 \%$ was obtained from Riedelde Haën. All chemicals were used as received.

Hydrogel synthesis

PNIPA gels were prepared by free radical crosslinking copolymerization in water, which is a solvent for all components of the mixture [26]. Proper amounts of monomers (NIPA and SA) and crosslinker (BIS) were dissolved in deionized water and then mixed with APS (initiator) water solution. The NIPA/water ratio was fixed at $1 / 10(\mathrm{wt} / \mathrm{wt})$, and the overall concentration of initiator (APS) and activator (TEMED) were 12.5 and $6 \mathrm{wt} \%$, with respect to NIPA content. The mixture was degassed and stirred at room temperature until all the solids dissolved completely. After dissolution of NIPA, the resultant solution was degassed with nitrogen for $10 \mathrm{~min}$. During degassing, the tube was immersed in an ice/water bath to minimize possible pre-polymerization. After addition of TEMED, gelation is achieved in less than $2 \mathrm{~min}$ at room temperature. To ensure completion of the copolymerization, the synthesized polymer was maintained for $24 \mathrm{~h}$ at room temperature. When the reaction was terminated, the gel was cut into small pieces and swollen in excess of deionized water thoroughly. During the following $48 \mathrm{~h}$, the swollen gel was washed several times with deionized water in order to remove unreacted monomers and linear polymer chains. The hydrogel was dried at room temperature, then in a vacuum oven at $70{ }^{\circ} \mathrm{C}$ to constant weight.

The preparation of the linear copolymer was similar to that of crosslinked copolymers as described above, but without addition of crosslinker.

Poly $(N$-isopropylacrylamide) gels containing different amounts of ionic comonomer (SA) and crosslinker (BIS) were synthesized. The amounts of SA were changed from 2 to $10 \mathrm{wt} \%$ and BIS from 2 to $20 \mathrm{wt} \%$. Table 1 shows the sample codes as well as compositions for NIPA/SA/BIS copolymer gels. The first number of the code indicates the percentage of crosslinker; the second number refers to the fraction of sodium acrylate.

Preparation of polymer/water mixtures

Gels were dried under vacuum for a few days at $70{ }^{\circ} \mathrm{C}$, after which the water content was less than $1.5 \mathrm{wt} \%$, as determined by thermogravimetric analysis. Polymer/water mixtures 
Table 1 Sample codes and compositions PNIPA

\begin{tabular}{ll}
\hline Code & NIPA/BIS/SA \% (wt/wt/wt) \\
\hline B0S0 & $100 / 0 / 0$ \\
B2S0 & $100 / 2 / 0$ \\
B0S5 & $100 / 0 / 5$ \\
B2S5 & $100 / 2 / 5$ \\
B5S5 & $100 / 5 / 5$ \\
B10S5 & $100 / 10 / 5$ \\
B15S5 & $100 / 15 / 5$ \\
B20S5 & $100 / 20 / 5$ \\
B2S10 & $100 / 2 / 10$ \\
B2S2 & $100 / 2 / 2$ \\
\hline
\end{tabular}

containing 70 to $95 \%$ of water were prepared by direct addition of the appropriate amount of water to the dried gels, then stored in refrigerator overnight. The homogeneous mixtures were encapsulated in aluminum hermetic pans for calorimetric analysis. Simultaneously, sample weight loss was measured by TGA to check the preparation procedure and determine the effective water content (error $\leq 1 \mathrm{wt} \%$ ).

\section{Gel permeation chromatography}

GPC measurement of uncrosslinked polymers (B0S0 and B0S5) was performed at $45^{\circ} \mathrm{C}$ in DMF with a nominal flow rate of $1 \mathrm{~mL} / \mathrm{min}$. A multiangle light scattering detector (DAWN EOS, Wyatt Technology, $\lambda=658 \mathrm{~nm}$ ), refractive index detector (Dn-2010 RI, WGE Dr. Bures), and column system (PSS, $100 \AA$, and two PL gel MIXED-C) were used. The results were evaluated using ASTRA 5.3.4.10 software from Wyatt Technologies and WINGPC 6.0 software from PSS. The refractive index increment $(\mathrm{dn} / \mathrm{dc})$ of PNIPAM in DMF equal to $0.075 \mathrm{~mL} / \mathrm{g}$ determined in separated experiment was used for calculations.

\section{Thermal analysis}

\section{Thermogravimetry}

Water content of the hydrogels was determined with a PerkinElmer Pyris Diamond TG/DT analyzer. Samples were placed in aluminum open sample pans (capacity $90 \mu \mathrm{L}$ ) and heated at $20{ }^{\circ} \mathrm{C} / \mathrm{min}$ from room temperature to complete evaporation of water. High-purity nitrogen gas was fluxed into the furnace at a flow rate of $50 \mathrm{~mL} / \mathrm{min}$.

As mentioned above, thermogravimetry was used to check the water content of the hydrogels, simultaneously with differential scanning calorimeter (DSC) analyses, as well as to determine eventual residual water in the dried polymers.

\section{Calorimetry}

Differential scanning calorimetry measurements were conducted with a Mettler DSC 30 calorimeter, Mettler Toledo, equipped with a liquid nitrogen cooling accessory, in order to determine the glass transition of the dried gels. Pure nitrogen was purged during the measurements. Dried $N$ isopropylacrylamide gels were heated in aluminum pans from room temperature to $250^{\circ} \mathrm{C}$ at $20^{\circ} \mathrm{C} / \mathrm{min}$. The glass transition was taken at the half height of changes of heat capacities between solid and liquid state.

Additional DSC analyses were also performed using a TAQ2000 differential scanning calorimeter equipped with a RCS-90 cooling unit (TA Instruments). The instrument was calibrated with pure indium. About 2-3 mg of the hydrolyzed gels were sealed into a Tzero ${ }^{\circledR}$ hermetic aluminum pan. The measurements were carried out from -50 to $100{ }^{\circ} \mathrm{C}$ at the heating rate of $20{ }^{\circ} \mathrm{C} / \mathrm{min}$, followed by cooling at $10{ }^{\circ} \mathrm{C} /$ min. High-purity nitrogen gas was fluxed at $20 \mathrm{~mL} / \mathrm{min}$ during all measurements and thermal treatments. No noticeable difference in sample mass before and after each DSC run was detected, indicating that no water evaporation took place during DSC analysis using the Tzero ${ }^{\circledR}$ hermetic aluminum pans.

\section{Results and discussion}

\section{Glass transition of dry gels}

The influence of crosslinker on the glass transition of dried poly( $N$-isopropylacrylamide) was determined by differential scanning calorimetry, and results are presented in Figs. 1 and 2. Figure 1 compares two series of the DSC curves. First series concerning dried PNIPA gels containing $5 \%$ of sodium acrylate and varied amounts of $N, N^{\prime}$-methylenebisacrylamide (BxS5). Second series illustrates the thermal properties of PNIPA gels containing $2 \%$ BIS and varied ionic comonomer content (B2Sx).

From this plot, the glass transition temperature $(\mathrm{Tg})$ is derived, and is plotted in Fig. 2 as function of BIS and SA content. $\mathrm{Tg}$ of linear PNIPA, not containing BIS or SA, is also shown in Fig. 2 for comparison.

The addition of crosslinker entails an increase in glass transition temperature of PNIPA, and the transition range becomes broader. This is to be linked to restrictions on PNIPA chain mobility in the gels, which increase with the amount of crosslinker [27]. The higher crosslinking density reduces the mobility of chain segments, so that the glass transition temperature increases. The varied broadness of the transition upon addition of crosslinker is to be related to a wider distribution of molecular weight between crosslinks at high BIS content [28]. 


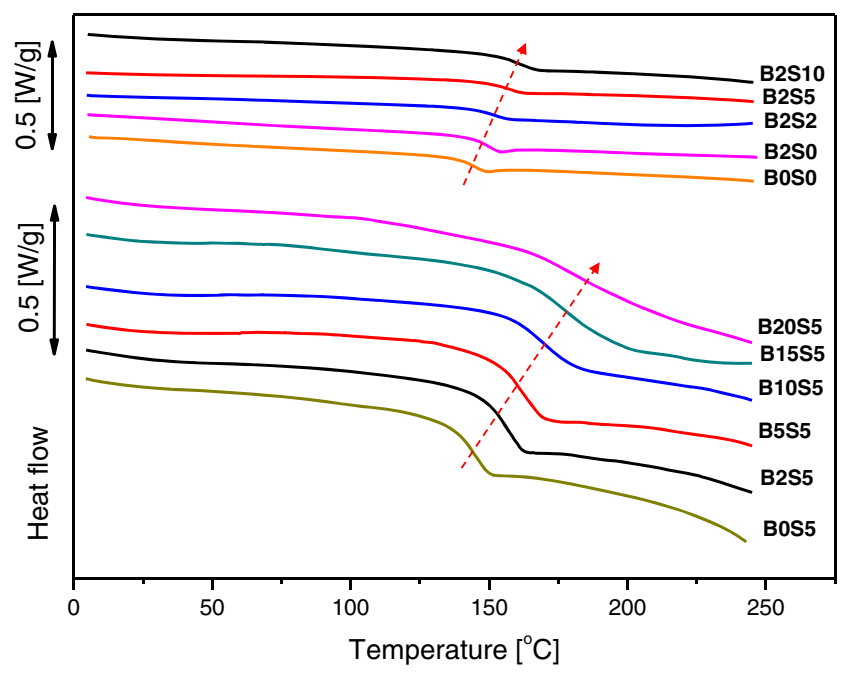

Fig. 1 DSC plots of PNIPA dry gels containing: $5 \%$ of sodium acrylate and varied amounts of $N, N^{\prime}$-methylenebisacrylamide; $2 \%$ of $N, N^{\prime}$ methylenebisacrylamide and varied percentage of sodium acrylate, upon heating at $20^{\circ} \mathrm{C} / \mathrm{min}$. The dashed arrow is a guide for the eyes

In order to determine whether the glass transition temperature is affected by chain length of the copolymer, the molecular mass of the two synthesized linear copolymers was determined by GPC. GPC analysis revealed that Mw of B0S0 is $731.8 \mathrm{kDa}$, whereas $\mathrm{Mw}=1,097 \mathrm{kDa}$ for B0S5. Molecular weight distribution $(\mathrm{Mw} / \mathrm{Mn})$ of both polymers is similar and equals 2.132 and 2.169 , respectively. These data indicate that both the linear polymers have high and comparable molecular mass, hence the increase in $T g$ upon introduction of $5 \mathrm{wt} \%$ of sodium acrylate is not caused by the difference in molecular mass of the two polymers [29].

As shown in Fig. 2, introduction of ionic comonomer in the structure of PNIPA has an effect similar to increase of network density, as progressive increase in ionic comonomer in PNIPA

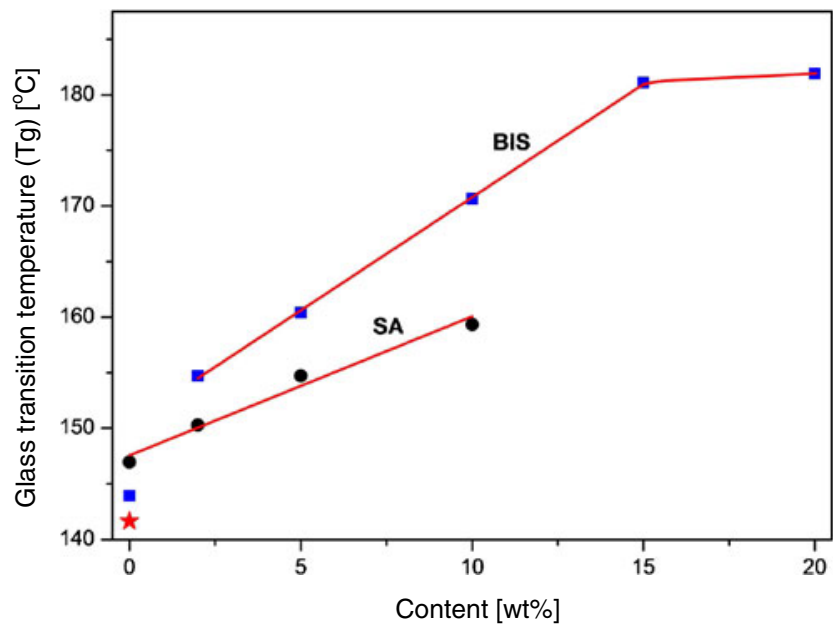

Fig. 2 Glass transition temperature ( $T g$ ) of dried PNIPA copolymers, as function of $N, N^{\prime}$-methylenebisacrylamide (BIS) content (square) and of sodium acrylate $(S A)$ content (circle). $T g$ of plain linear PNIPA ( $\downarrow \mathbf{\Lambda}$ ) is also reported for comparison formulation produces a corresponding increase of $T g$, which moves to higher temperature with SA fraction. This indicates that the presence of sodium acrylate induces a higher rigidity of the polymer network, at parity of crosslinker content.

Thermal analysis presented above could be extended to more quantitatively to obtain more information about an origin of behavior based on molecular motion in the glass transition area such as it was early performed for different polymers and copolymers in literature [30-32]. Unfortunately, we are not able to present in this paper the quantitative thermal analysis of glass transition of our polymeric material, PNIPA copolymer without first an establishing the series of equilibrium of thermodynamic parameters and functions. This kind of task should be an aim of near future studies.

Demixing/mixing transition of PNIPA hydrogels — influence of ionic comonomer

As mentioned above, polymer gels, consisting of an uncrosslinked or crosslinked polymer network immersed in water, undergo a demixing/mixing transition upon variation of temperature. The influence of PNIPA gel composition on this transition was analyzed as a function of water swelling. Figure 3 shows an example of heat-flow-rate vs. temperature plot for the 100/5 wt/wt linear copolymer (B0S5). The plots refer to the hydrogel swollen with $90 \mathrm{wt} \%$ of water. The main transitions detected in the heating scan are an endothermic peak around $0{ }^{\circ} \mathrm{C}$ due to water melting, and an endothermic peak at $29.0^{\circ} \mathrm{C}$ due to demixing. Mixing is revealed as an exotherm around $29.2{ }^{\circ} \mathrm{C}$ in the subsequent cooling. Furthermore during cooling, crystallization of water takes

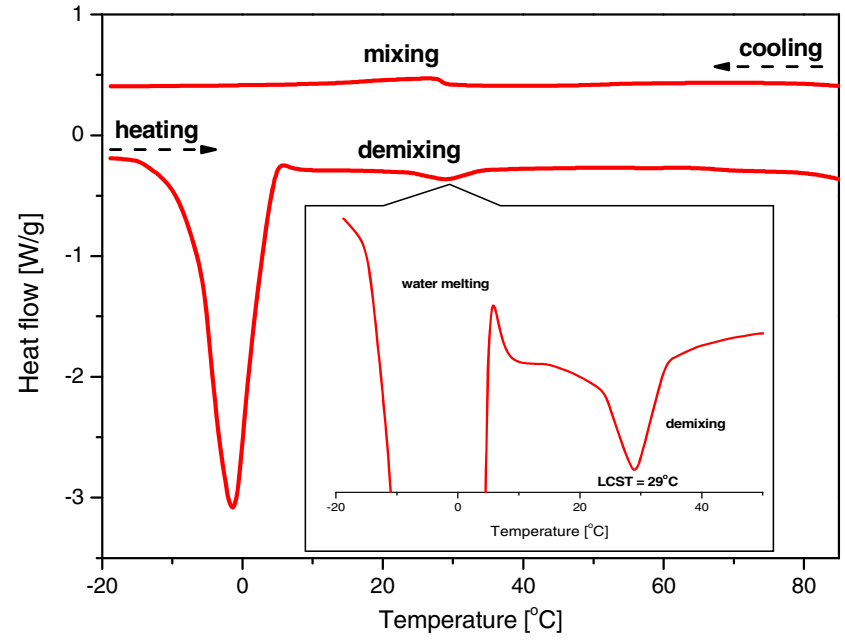

Fig. 3 DSC thermogram of sample copolymer PNIPA (the gel contains $5 \%$ of sodium acrylate, B0S5) in equilibrium swelling degree with $90 \%$ of water; cooling rate, $10^{\circ} \mathrm{C} / \mathrm{min}$; heating rate (second run), $5{ }^{\circ} \mathrm{C} / \mathrm{min}$. The inset illustrates an enlargement of the DSC plot to show details of the demixing transition 


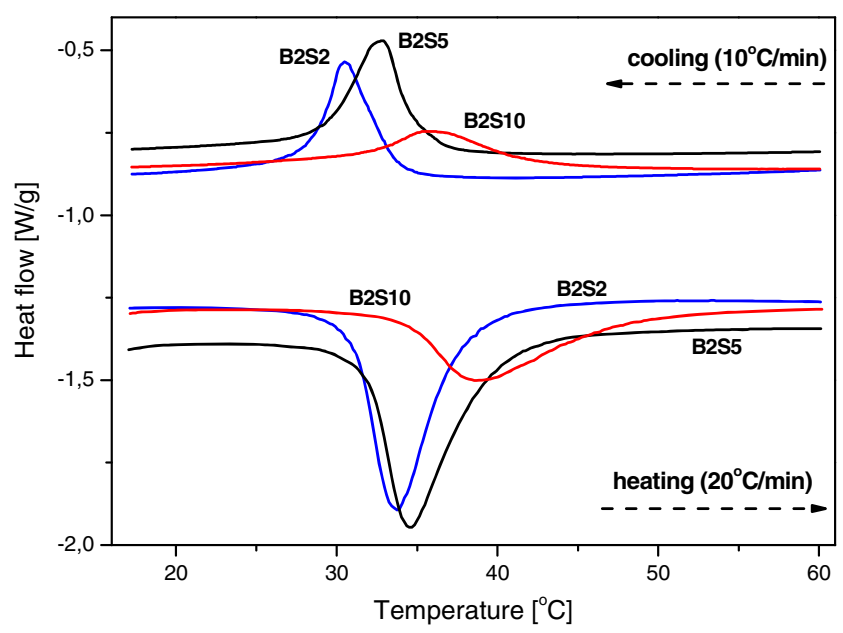

Fig. 4 Demixing/mixing DSC plots of PNIPA hydrogels swollen with $75 \%$ of water. The gel contains $2 \%$ of $N, N^{\prime}$-methylenebisacrylamide and varied amounts of sodium acrylate

place, but this is not shown in Fig. 3. Crystallization exothermic peak is very intensive and then other peaks (demixing and mixing) are not visible.

Figure 4 shows a few examples of the DSC traces, gained upon heating and cooling, of one selected hydrogel/water ratio $(25 / 75 \mathrm{wt} / \mathrm{wt} \%)$, where the PNIPA copolymers contain varied amounts of sodium acrylate. The endothermic demixing, seen in Fig. 4 upon heating, becomes broader and moves to higher temperatures with the increase of sodium acrylate content. Similarly, upon cooling higher amounts of SA correspond to an earlier occurrence of demixing. It is worth to note that at low SA content, not only the mixing transition is narrower, but the exothermic peak has a sharper onset. The faster initiation of the transition, as well as the reduced temperature range where it occurs, is probably due to a more homogeneous network structure at low SA content [33].

Figure 5 presents the phase diagrams measured upon heating and cooling of the PNIPA/water hydrogels containing $2 \%$ of BIS, where the LCST is plotted as function of gel content, in the range $5-30 \mathrm{wt} \%$ of gel in water. The onset points, derived from the DSC traces as intercepts between the baseline before the transition and the inflectional tangent, were taken into account to build the phase diagram, to quantify the beginning of phase separation and coalescence upon heating and cooling, respectively. The LCST varies with gel composition, and occurs at higher temperatures for gels containing higher amounts of sodium acrylate. The mixing temperatures are only slightly affected by water content, whereas the demixing transition displays a somewhat more marked dependence on water swelling, as higher amounts of water result in a higher temperature of demixing, in the analyzed hydrogel composition range.

The varied composition dependence of mixing and demixing transitions is probably to be linked to the kinetics

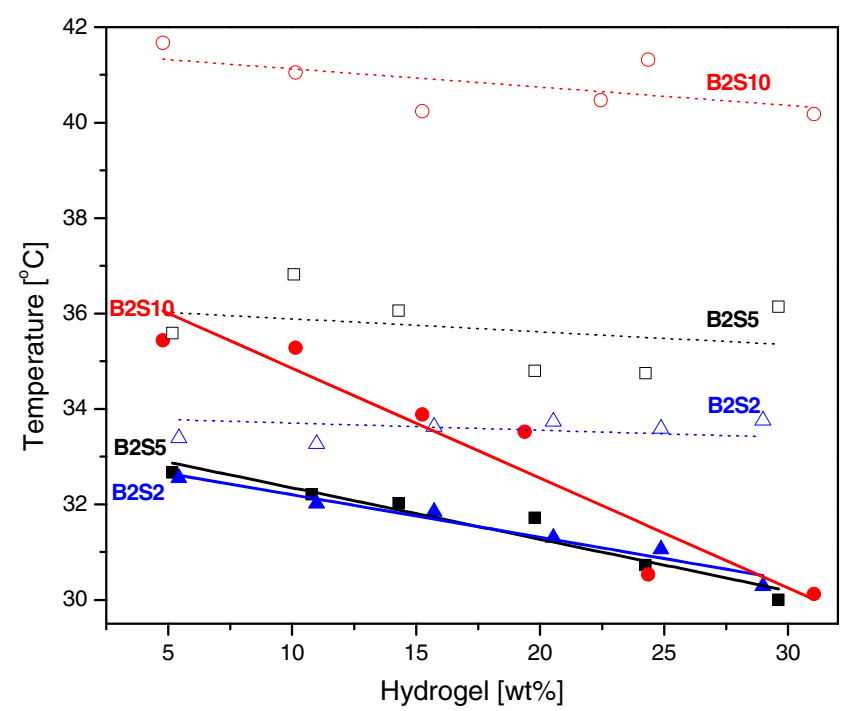

Fig. 5 LCST (onset temperature) of the demixing/mixing transition of PNIPA hydrogels containing $2 \%$ of $N, N^{\prime}$-methylenebisacrylamide and varied percentage of sodium acrylate, as function of polymer content. Data were measured upon heating at $20^{\circ} \mathrm{C} / \mathrm{min}$ (close symbols), and cooling at $10{ }^{\circ} \mathrm{C} / \mathrm{min}$ (open symbols)

of the two processes. As reported in the literature, the thermoshrinking type of demixing of hydrogels in water might be induced through an aggregation of polymer segments due to hydrophobic interaction [34]. Generally, the strength of the hydrophobic interaction is proportional to the number of water molecules that form the hydrophobic hydration and increases with temperature. It can therefore be hypothesized that the gel whose hydrophobic groups have a larger surface (contact) area undergo a discontinuous demixing in water at lower temperatures due to the strength of the hydrophobic interaction [34]. This clarifies the effect of sodium acrylate, which is an ionic comonomer and hence increases hydrophilic character of the polymer network, on demixing of PNIPA hydrogels.

The heat associated with the demixing of the hydrogel is also affected by comonomer content, as seen in Fig. 6, which displays the enthalpy of demixing of the hydrogels containing different amounts of SA as function of polymer content.

At parity of water/polymer ratio, increasing content of ionic comonomer causes a decrease of the demixing enthalpy. Increasing of ionization degree of the gel results in a growth of interactions between polymer chains and a decrease of polymer-water interactions. This implies that lower energy is needed to detach water from polymer and the demixing enthalpy reduces. In turn, increasing amounts of water (decreasing amounts of hydrogel) result in a lower exchange of latent heat upon demixing transition regardless of ionic comonomer content. Direct polymer-water interaction involves only a limited fraction of water molecules, so that not all water molecules interact with polymer chains [35]. Therefore increasing amount of water in polymer-water 


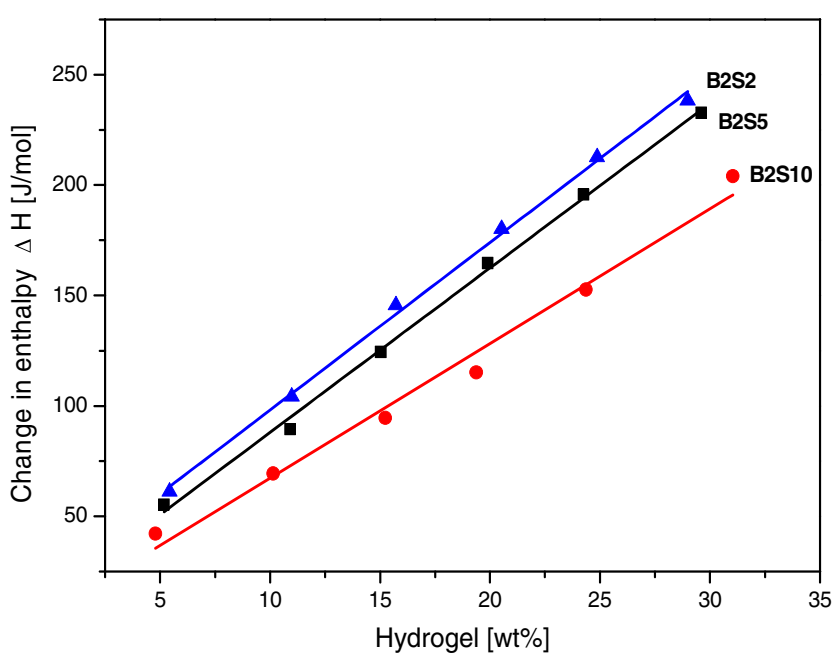

Fig. 6 Demixing enthalpy of PNIPA hydrogels containing $2 \%$ of $N, N^{\prime}$ methylenebisacrylamide and varied percentage of sodium acrylate, as function of polymer content

system causes decrease of the demixing enthalpy, at parity of hydrogel mass.

Demixing/mixing transition of PNIPA hydrogels—influence of crosslinker

The DSC thermograms in the region of demixing/mixing transition of PNIPA hydrogels containing $5 \%$ of sodium acrylate and varied percentages of crosslinker are presented in Fig. 7.

The plots, measured first upon cooling, then on subsequent heating, refer to hydrogels swollen with $75 \%$ of water. Similar plots were gained also for gels swollen with different amounts of water, ranging from 70 to $95 \%$. The onset temperatures of

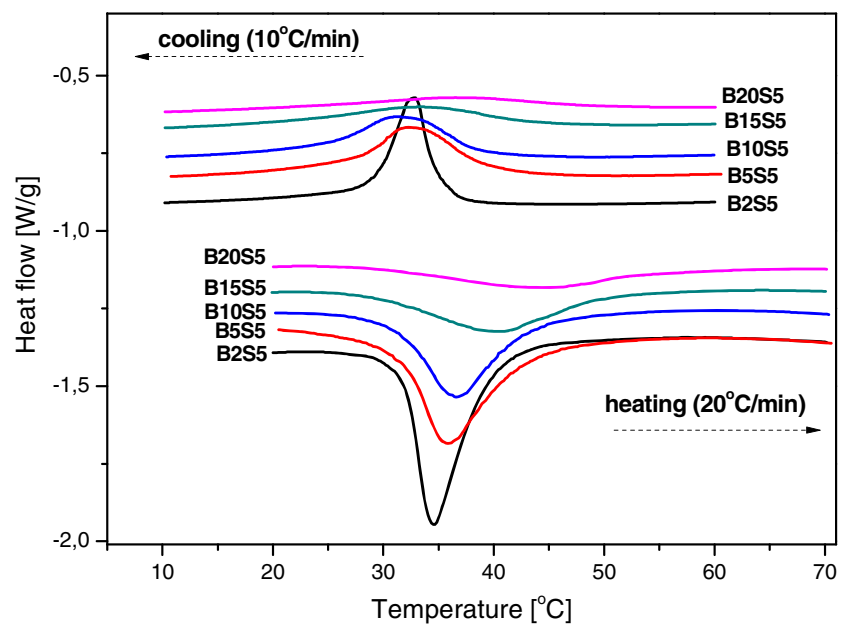

Fig. 7 Demixing/mixing transition plots by DSC of PNIPA hydrogels swollen with $75 \%$ of water. The dry gel contains $5 \%$ of sodium acrylate and varied amounts of $N, N^{\prime}$-methylenebisacrylamide (BXS5, where $X=$ $2,5,10,15,20)$ mixing and demixing, determined from the DSC data, are reported in Fig. 8.

Crosslinked hydrogels, as well as the linear polymer, do not show significant changes of LCST with water content, in the analyzed PNIPA/water composition range. The slight decrease of demixing point with water content is close to the experimental uncertainty associated to DSC analysis, as also probed by the scattering of the data. For the linear polymer (B0S5), instead, the LCST markedly increases with water content upon heating, whereas the onset of mixing is not affected by polymer/water ratio. On the other hand, the LCST increases quite regularly with increasing degree of crosslinking upon cooling. These changes are not so unequivocal upon heating. This lack of regular changes of LCST values with change of water content can be explain also by the shape of phase diagrams of particular gel/water systems. This problem will be discussed in a separate paper.

a

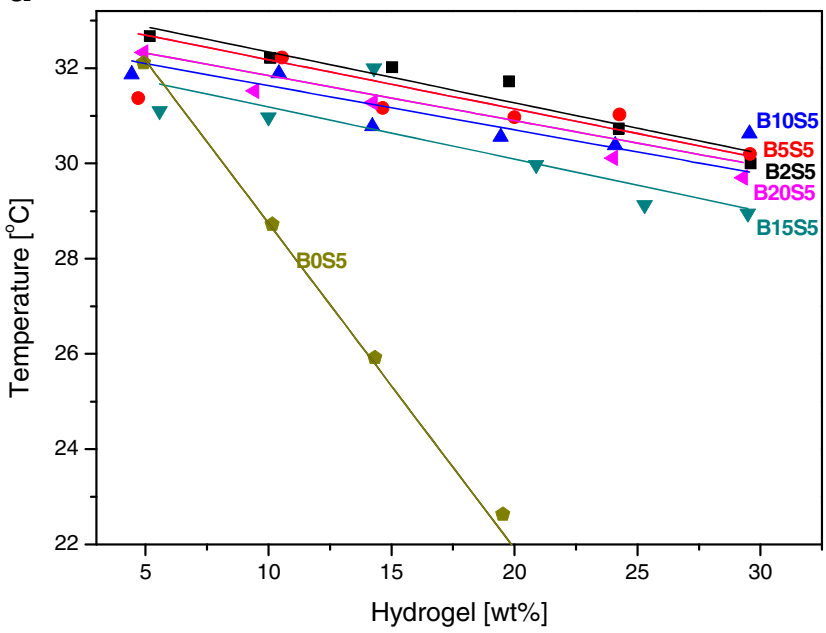

b

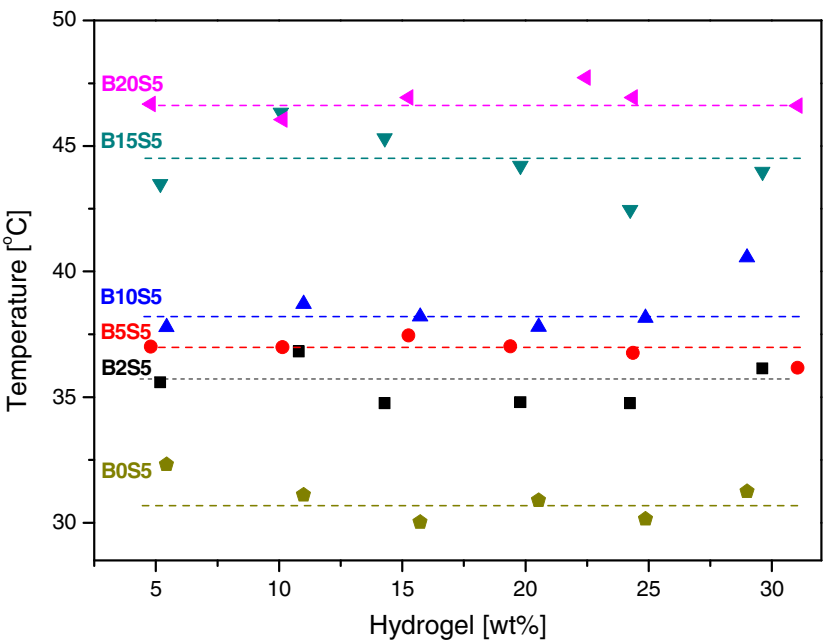

Fig. 8 Onset temperature of the demixing/mixing transition of PNIPA hydrogels containing $5 \%$ of sodium acrylate and varied percentage of $N$, $N^{\prime}$-methylenebisacrylamide, as function of polymer content, data measured: a upon heating at $20{ }^{\circ} \mathrm{C} / \mathrm{min}$, b upon cooling at $10{ }^{\circ} \mathrm{C} / \mathrm{min}$ 
The mixing enthalpy of PNIPA hydrogels containing $5 \%$ of sodium acrylate and varied percentage of $N, N^{\prime}$ methylenebisacrylamide, is exhibited in Fig. 9 as function of polymer content. The enthalpy of transition linearly varies with the PNIPA/water ratio, as seen in Fig. 9. Moreover, an increase of crosslinker results in a broader and weaker transition, as the latent heat associated to demixing transition decreases with BIS content.

The demixing enthalpy quantifies the effective waterpolymer interactions, which depend on degree of crosslinking. At fixed amount of water, polymer-water interactions are stronger if the content of crosslinker is in the range 0 $5 \mathrm{wt} \%$, compared to the PNIPA formulations containing 10 $20 \mathrm{wt} \%$ of crosslinker. At low BIS content $(0-2 \mathrm{wt} \%)$, the surface (contact) area of polymer-water increases and it results in a growth of the demixing enthalpy. Further increase of BIS content in the polymer causes a decrease of the demixing enthalpy. A high degree of crosslinking (BIS content $\geq 10 \mathrm{wt} \%$ ) makes the gel structure more compact, with decreased interactions between polymer and water. On the other hand, the demixing enthalpy decreases with the increase of water content in polymer/water system. The slope of enthalpy vs. composition curves is also affected by BIS content, and becomes less steep with progressive increase of crosslinking degree of the polymer [36, 37].

As detailed above, higher amounts of crosslinker lead to an increased rigidity of the polymer network structure. This in turn, may affect the thermoshrinking transition of PNIPA hydrogel, which occurs at lower temperature in gels that have higher chain mobility. The crosslinker density affects also the enthalpy of transition, which decreases in the hydrogels that have higher rigidity of the polymer network. The increased breadth of the transition is to be linked to the wider

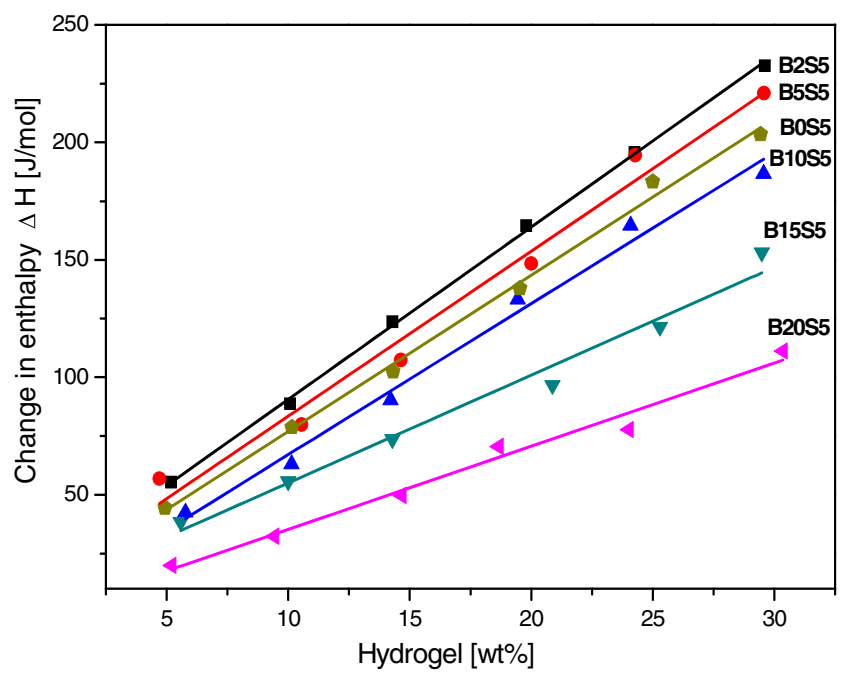

Fig. 9 Demixing enthalpy of PNIPA hydrogels containing $5 \%$ of sodium acrylate and varied percentage of $N, N^{\prime}$-methylenebisacrylamide, as function of polymer content distribution of molecular weight between crosslinks at high BIS content.

\section{Conclusions}

The reported results clarify the effect of introduction of sodium acrylate as ionic comonomer on the thermal properties of PNIPA-SA copolymers, as well as the influence of crosslinking density on glass transition of these copolymers and on the mixing/demixing transitions. Inclusion of sodium acrylate as ionic comonomer into thermosensitive poly $(N$ isopropylacrylamide) causes an increase of the glass transition temperature of this new copolymer. An increase of the crosslinking degree of PNIPA-SA copolymer also results in higher temperatures of glass transition, which also takes place in a wider temperature range.

The higher crosslinking density and higher ionization level of PNIPA reduces the mobility of chain segments and at the same time induces a higher rigidity of the polymer network, what shifts glass transition temperature to higher values. It was found that in case of both the linear polymers, molecular mass does not affect the glass transition temperature. Therefore an enhancement of glass transition temperature is caused only by increased network density, which is a result of higher content of the crosslinker or the ionic comonomer.

When the gel is swollen with water, composition of the PNIPA-SA copolymer affects also the LCST. In hydrogels containing higher amounts of ionic comonomer, mixing/ demixing occur at higher temperature, at parity of polymer/ water ratio. A similar effect is seen upon variation of crosslinking density of the polymer swollen with water, since increasing amounts of BIS result in a higher mixing temperature.

The demixing of hydrogels/water system is induced by an aggregation of polymer segments due to hydrophobic interaction. Increasing content of sodium acrylate (ionic comonomer) influence on demixing of PNIPA hydrogels by an increase of hydrophilic character of the polymer network. Hydrophobic interactions are proportional to the number of water molecules that form the hydrophobic hydration and increase with temperature. These gels, which hydrophobic groups have a smaller contact area undergo a discontinuous demixing in water at higher temperature.

Similarly, the higher amounts of crosslinker increases the rigidity of the polymer network structure and results in appearance of the thermoshrinking transition of PNIPA hydrogel at higher temperature.

The demixing enthalpy increases together with the decrease of the water content in the gel. The enthalpy decreases with the increase of the ionization degree at determined crosslinking degree of the gel. Increasing of ionization degree of the gel results in a growth of interactions 
between polymer chains and a decrease of polymer-water interactions. This implies that lower energy is needed to detach water from polymer and the demixing enthalpy reduces. On the other hand, increasing amounts of water result in a lower exchange of latent heat upon demixing transition regardless of ionic comonomer content. Direct polymer-water interaction involves only a limited fraction of water molecules, therefore increasing amount of water in polymerwater system causes decrease of the demixing enthalpy, at parity of hydrogel mass.

At the defined content of ionic comonomer, the demixing enthalpy initially increases with increasing amount of crosslinker and after exceeding a certain degree of crosslinking ( $2 \mathrm{wt} \%$ crosslinker content) it decreases.

The demixing enthalpy quantifies the effective waterpolymer interactions, which depend on degree of crosslinking. At low BIS content, the contact area of polymer-water increases and it results in a growth of the demixing enthalpy. Further increase of BIS content in the polymer causes a decrease of the demixing enthalpy. A high degree of crosslinking makes the gel structure more compact, with decreased interactions between polymer and water. In turn, the demixing enthalpy decreases with the increase of water content in polymer/water system. The demixing enthalpy decreases with the increase of water content in polymer/ water system.

Open Access This article is distributed under the terms of the Creative Commons Attribution License which permits any use, distribution, and reproduction in any medium, provided the original author(s) and the source are credited.

\section{References}

1. Schild H (1992) Prog Polym Sci 17:163-249

2. Qiu Y, Park K (2001) Adv Drug Rev 53:321-339

3. Aoyagi T, Ebara M, Sakai K, Sakurai Y, Okano T (2000) J Biomater Sci Polym Ed 1:101-110

4. Aoki T, Muramatsu M, Torii T, Sanui K, Ogata N (2001) Macromolecules 34:3118-3119
5. Suwa K, Yamamoto K, Akashi M, Takano K, Takana N, Kunugi S (1998) Colloid Polym Sci 276:529-553

6. Madea Y (2001) Langmuir 17:1737-1742

7. Pelton R (2000) Adv Colloid Interface Sci 85:1-33

8. Gil E, Hudson S (2004) Prog Polym Sci 29:1173-1222

9. Galaev I, Mattiasson B (1999) Trends Biotech 17:335-340

10. Hoffman A, Stayton P (2004) Macromol Symp 207:139-151

11. Lutz H (2006) Polym Int 55:979-993

12. Hoare T, Kohane D (2008) Polymer 49:1993-2007

13. Kost J, Langer R (2001) Adv Drug Deliv Rev 46:125-148

14. Sassi A, Shaw A, Han S, Blanch H, Prausnitz M (1996) Polymer 37: 2151-2164

15. Tokuyama H, Kanehara A (2007) Reactive \& Funct Polym 67:136143

16. Dong L, Hoffaman A (1991) J Controlled Release 15:141-152

17. Kraftz K, Hellweg T, Eimer W (2000) Colloide Surf A 170:137-149

18. Van Drume K, Van Assche G, Van Mele B (2004) Macromolecules 37:9596-9605

19. Haraguchi K, Takehisa T, Fan S (2002) Macromolecules 35:1016210171

20. Percot A, Zhu X, Lafleur M (2000) J Polym Sci: Part B: Polym Phys 38:907-915

21. Erbil C, Kazancıoğlu E, Uyanık N (2004) Europ Polym J 40:11451154

22. Xue W, Champ S, Malcolm B (2000) Polymer 41:7575-81

23. Liu Y, Velada J, Huglin M (1999) Polymer 40:4299-306

24. Matsuo E, Tanaka TJ (1988) Chem Phys 89:1695-7

25. (1996) Patent EP 0693508 A1

26. Muca R, Zarzyka I, Piątkowski W, Antos D (2010) Thermosensitivegels with fast temperature response for low-pressure chromatography of proteins, SPICA 2010, Stockholm, Sweden, p 2031

27. Montserrat SJ (1993) Therm Anal Calorim 40:553-563

28. Haraguchi K, Takehisa T, Fan S (2002) Macromolecules 35:1016210171

29. Blanchard L-P, Hesse J, Malhotra S (1974) Can J Chem 52:3170 3175

30. Buzin A, Pyda M, Matyjaszewski K, Wunderlich B (2002) Polymer 43:5563-5569

31. Pyda M, Van Durme K, Wunderlich B, Van Mele B (2005) J Polymer Sci, Part B: Polymer Phys 43:2141-2153

32. Di Lorenzo ML, Pyda M, Wunderlich B (2001) J Polymer Sci: Part B: Polymer Phys 39:1594-1604

33. Ni C, Zhu X-X (2004) Europ Polym J 40:1075-1080

34. Inomata H, Goto S, Saito S (1990) Macromolecules 23:4887-4888

35. Coronado R, Pekerar S, Lorenzo AT, Sabino MA (2011) Polym Bull 67:101-124

36. Bueno V, Bentini R, Catalani L (2013) Carbohydr Polym 92:10911099

37. Gulrez S, Al-Assaf S, Phillips G (2011) Progress in molecular and environmental bioengineering - from analysis and modeling to technology applications. InTech Publisher, Rijeka, pp 117-150 